\title{
Towards Realistic Implementations of Large Imaging Calorimeters
}

\section{Lorenz Emberger*†}

Max-Planck-Institute for Physics

E-mail: emberger@mpp.mpg.de

\begin{abstract}
The next generation of collider detectors will most likely make full use of Particle Flow algorithms, requiring precision tracking and imaging calorimeters. The latter, with granularity 2 to 3 orders of magnitude above existing devices, have been developed during the last 15 years by the CALICE collaboration and are now approaching maturity. The state-of-the-art and the remaining challenges will be presented for all the investigated readouts: silicon diodes and scintillator for a tungsten electromagnetic calorimeter, gaseous with semi-digital readout and scintillator with SiPM readout for a hadronic one. We will describe the commissioning, including beam tests, of large scale technological prototypes of a silicon tungsten electromagnetic calorimeter and hadron calorimeters featuring either a gaseous medium or scintillator with SiPM as an active material. Where applicable, raw performances of calorimeter such as energy resolution and linearity will be presented but also studies exploiting the distinct features of granular calorimeters regarding pattern recognition. Beyond these prototypes, the design of experiments addressing the requirements and potential of imaging calorimetry will be commented on. In addition, less established but promising techniques for dedicated devices will be highlighted.
\end{abstract}

European Physical Society Conference on High Energy Physics - EPS-HEP2019 -

10-17 July, 2019

Ghent, Belgium

\footnotetext{
*Presenter

${ }^{\dagger}$ On behalf of the CALICE collaboration
} 


\section{Introduction}

To fully expolit the potential of future $\mathrm{e}^{+} \mathrm{e}^{-}$collider experiments, a precise reconstruction of all final states is necessary. To achieve this for hadronic final states, a jet energy resolution significantly beyond the current state-of-the-art, in the order of $3 \%$ - $4 \%$ over a wide energy range, is required. Such a resolution, which would provide the capability to separate hadronically decaying $\mathrm{W}^{ \pm}$and $\mathrm{Z}$ bosons, requires specialised detectors and reconstruction algorithms. In contrast to traditional energy measurement of summing up the energy depositions in all sub-detectors, the Particle Flow (PF) approach is designed to measure different types of particles of a jet in the best suited detector. For charged particles, the tracker typically offers the best energy resolution. Photon energies are measured in the electromagnetic calorimeter (ECAL) and the energy of neutral hadrons is measured in the hadronic calorimeter (HCAL). Since charged particles and photons carry about 90\% of the energy in a typical jet, particle flow is intrinsically increasing the jet energy resolution. Only the remaining $10 \%$ jet energy carried by neutral hadrons is measured in the HCAL, which typically offers a worse energy resolution. To reduce double counting of charged particles in the tracker and calorimeter,it is vital to separate particle showers in the calorimeters and assign them to tracks. With increasing energies, the hadronic jets get narrower and the danger of "confusion" increases, transforming the energy measurement in a pattern recognition problem [7]. The CALICE collaboration is focussed on the design and realization of such systems. This contribution introduces the calorimeter prototypes developed by CALICE in section 2 and touches on the performance evaluated in extensive beam test campaigns in section 3. In section 4, developments towards compact electronics and up-scalability to large detector modules are presented.

\section{Prototypes of the CALICE Calorimeters}

The concepts considered in this contribution are two analog ECAL prototypes with tungsten absorbers and two HCAL prototypes, an analog iron-scintillator calorimeter and a digital gaseous calorimeter with RPC readout. These prototypes are called technological prototypes because they address constraints of realistic detector scenarios like compactness, power consumption and structural integrity over large areas. They are the successors of the physics prototypes which succesfully established the concept of highly granular calorimeters. The techonlogical prototypes feature power pulsing capability switching the electronics on and off in syncronization with the collision of a bunch train in a linear collider. This reduces power consumption and mitigates heat production inside the fiducial volume of the detector. A common family of read out ASICs (HARDROC, SPIROC, SKIROC) is used in CALICE to facilitate combined running and data acquisition which is already used in combined beam tests of ECAL and HCAL. The combined datasets are used to develop and test novel event reconstruction techniques in the scope of a full collider detector concept. Automated assembly techniques are being developed for all concepts to enable large scale production of modules in reasonable time scales.

\subsection{Electromagnetic Calorimeters}

A scintillator ECAL (Sc-ECAL) [3], which is currently under development, features crossed scintillator strips with an effective granularity of $5 \times 5 \mathrm{~mm}^{2}$ interleaved with metal absorbers. The 
strips are read out by surface mounted silicon photomultipliers (SiPM). The final prototype will consist of 30 layers.

The silicon tungsten ECAL (SiW-ECAL) [2] prototype consists of 15 subsequent layers of tungsten absorber and $9 \times 9 \mathrm{~cm}^{2}$ silicon wafers each hosting $5.5 \times 5.5 \mathrm{~mm}^{2}$ pixels. Despite the limited number of layers, this prototype successfully demonstrates the technological developments towards an integration in a full collider detector.

\subsection{Hadronic Calorimeters}

The analog hadronic calorimeter (AHCAL) [6] consists of 39 successive layers of a $17 \mathrm{~mm}$ thick steel absorber and a $3 \mathrm{~mm}$ thick scintillator as active material resulting in a depth of $\sim 6 \lambda$. The active material is segmented in $3 \times 3 \mathrm{~cm}^{2}$ tiles to achieve high granularity. This "SiPM-on-tile" technology features tiles which are individually wrapped in reflective foil and mounted directly on a printed circuit board hosting a SiPM. A full prototype was assembled in 2017, with high degree of automation in testing, component preparation such as wrapping of scintillator tiles, and module assembly.

The gaseous semi-digital hadronic calorimeter (SDHCAL) [1] features 48 layers of steel absorbers interleaved with $1 \mathrm{~m}^{2}$ Glass Resistive Plate Chambers (GRPC) as active medium. The GRPCs charge is collected by $1 \mathrm{~cm}^{2}$ copper electrodes and the read out is operated in 2 bit mode resulting in 3 thresholds. This detector was the first fully assembled and tested technological prototype with a depth $6 \lambda$.

\section{Performance in Beam Tests}

The CALICE Prototypes are extensively tested in beam test facilities recording muon, electron and hadron data. Due to the high spatial granularity it is possible to record images of electromagnetic and hadronic showers as shown in fig. 1 on the left. This feature enhances the capability of the calorimeters to separate close by showers, an essential feature of PF detectors. The single cell timing capability of the prototypes is a key feature of future collider detectors where high luminosity and beam-induced backgrounds cause increased pile up of background events. Another essential requirement for $\mathrm{PF}$ is the ability to trigger on and read out small energy depositions demanding a sufficient signal to noise $(\mathrm{S} / \mathrm{N})$ ratio. The right part of fig. 1 shows a noise peak around 3 ADC counts alongside a MIP measurement around 60 ADC in the SiW-ECAL. The resulting S/N of 20 [2] allows to trigger on energy depositions of a fraction of a MIP. Combined beam tests of electromagnetic and hadronic calorimeters successfully exploited the features of the common ASIC family to take data with a common DAQ system.

\section{Recent Mechanical and Electronic Developments}

The main purpose of the technological prototypes is the development and testing of methods to integrate the detectors in large collider detector systems, which require to meet spatial constraints as well as power and cooling limits while keeping the performance. The scalability of the comparably small prototypes of about $1 \mathrm{~m}^{2}$ front face area to complete barrel and endcap modules has to be established. As an example, the RPC plates of the SDHCAL rely on a homogeneous gas flow 

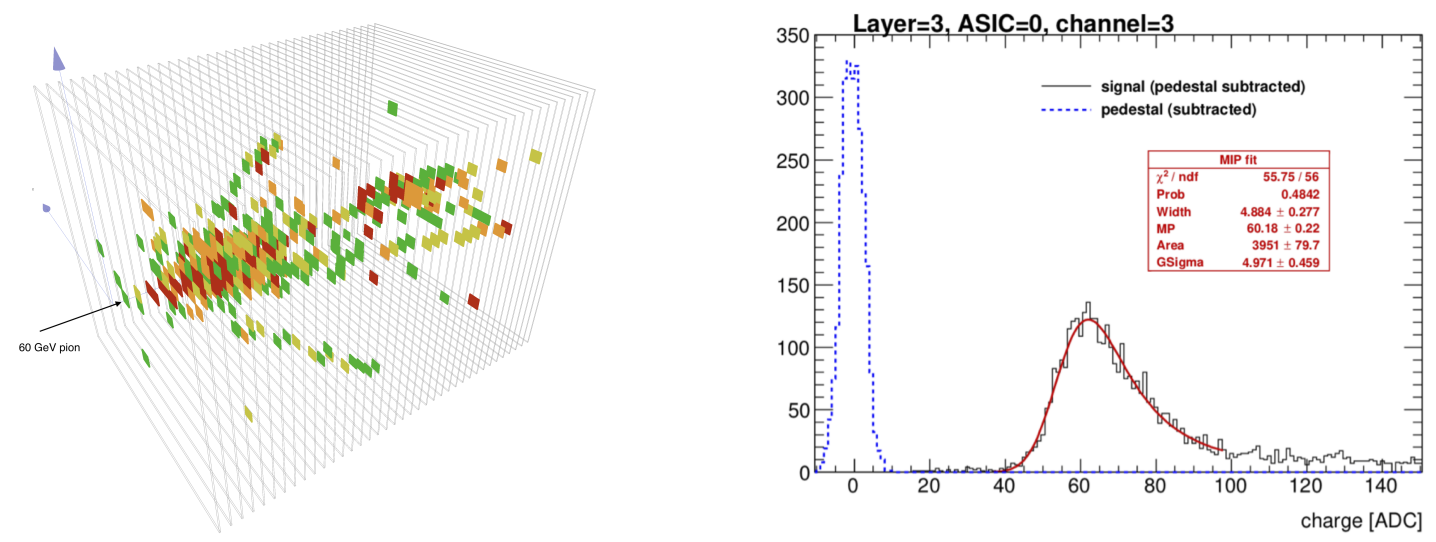

Figure 1: On the left, an image of a $60 \mathrm{GeV}$ pion hadronic shower in the AHCAL is shown. Red indicates high energy deposition, green indicates low energy deposition. On the right, a distribution of noise signals around 3 ADC counts is shown alongside the distribution of MIP energy depositions in the SiW-ECAL [2]. This results in a signal to noise ratio of 20 .

throughout the whole volume. A sophisticated gas circulation system is shown on the left side of fig. 2 providing a homogeneous gas distribution and volume exchange over a $2 \mathrm{~m}^{2}$ area. To distribute the power for the detector over these large areas in confined spaces, a powerful but compact electronic system is needed. As interfaces of the calorimeter subsystem to the data acquisition of a full collider detector these components are located outside of the fiducial detector volume. The right side of fig. 2 shows the detector interface (DIF) and power supply boards at the end of a more than $2 \mathrm{~m}$ long AHCAL module. The stable behaviour of the setup allows the assembly of full size detectors. Similar developments also exist for the SiW-ECAL technology. Furthermore, means of constructing precisely aligned absorber structures to provide rigid structural components for the calorimeters active layers are investigated.
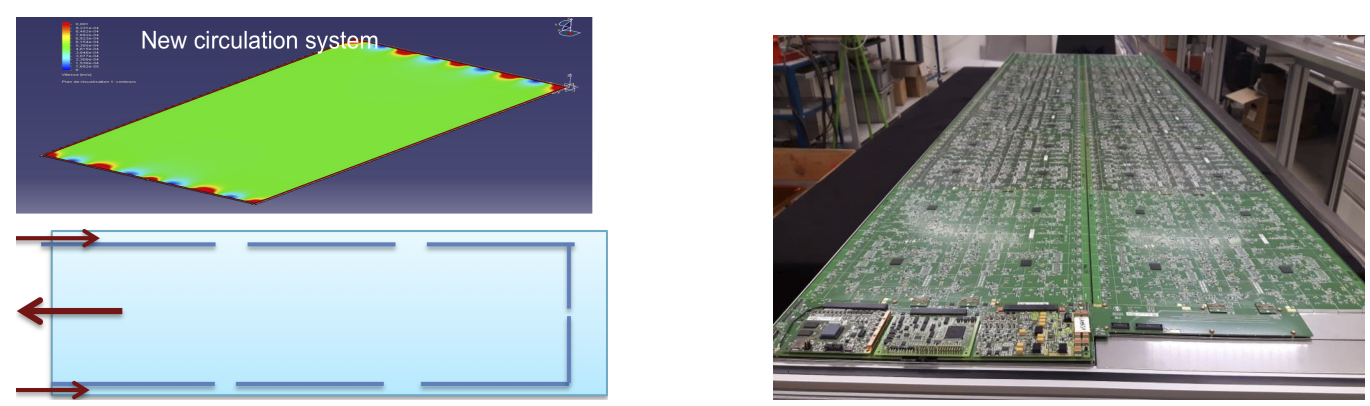

Figure 2: (Left: Improved gas distribution scheme in the RPC of the SDHCAL ensuring a homogeneous velocity profile of the gas flow. Right: Detector interface (DIF) and power supply boards of the AHCAL powering and reading a more than 2 meter long detector module containing 1728 channels. 


\section{Conclusion}

The calorimeters developed by CALICE are optimized for Particle Flow event reconstruction for future $\mathrm{e}^{+} \mathrm{e}^{-}$colliders. Extensive beam tests of the various prototypes validated the capabilities of the calorimeters and efforts towards up scalability led to compact but sophisticated read out electronics to operate large detector modules. The possibility of combined data taking facilitated the recording of an extensive dataset of electromagnetic and hadronic showers to develop and test novel event reconstruction algorithms. The expertise of CALICE is already applied in detector upgrades of the upcoming HL-LHC e.g. the CMS HGCAL [5] and the ALICE FOCAL [4].

\section{References}

[1] Guillaume Baulieu et al. "Construction and commissioning of a technological prototype of a high-granularity semi-digital hadronic calorimeter". In: Journal of Instrumentation 10 (June 2015). DOI: $10.1088 / 1748-0221 / 10 / 10 /$ P10039.

[2] S. Bilokin et al. "Commissioning of the highly granular SiW-ECAL technological prototype". In: (2018). arXiv: 1810.05133 [physics .ins-det ] .

[3] K. Francis et al. "Performance of the first prototype of the CALICE scintillator strip electromagnetic calorimeter". In: Nucl. Instrum. Methods Phys. Res., A 763.arXiv:1311.3761 (Nov. 2013). Preliminary entry, 278-289. 12 p. DOI: $10.1016 /$ j. nima. 2014.06 .039 . URL: http://cds.cern.ch/record/1629400.

[4] A.P. de Haas et al. "The FoCal prototype - an extremely fine-grained electromagnetic calorimeter using CMOS pixel sensors". In: Journal of Instrumentation 13.01 (Jan. 2018), P01014P01014. DOI: 10 . 1088/1748-0221/13/01/p01014. URL: https://doi .org/ $10.1088 \div 2 \mathrm{~F} 1748-0221 \div 2 \mathrm{~F} 13 \div 2 \mathrm{~F} 01 \% 2 \mathrm{Fp} 01014$.

[5] Christophe Ochando. "HGCAL: A High-Granularity Calorimeter for the endcaps of CMS at HL-LHC”. In: J. Phys. : Conf. Ser. 928.1 (2017), 012025. 4 p. DOI: 10 . $1088 / 1742-$ 6596/928/1/012025. URL: https://cds. cern.ch/record/2311394.

[6] Felix Sefkow and Frank Simon. "A highly granular SiPM-on-tile calorimeter prototype”. In: Journal of Physics: Conference Series 1162 (Jan. 2019), p. 012012. ISSN: 1742-6596. DOI: 10.1088/1742-6596/1162/1/012012. URL: http://dx.doi.org/10.1088/ $1742-6596 / 1162 / 1 / 012012$.

[7] Felix Sefkow et al. "Experimental Tests of Particle Flow Calorimetry". In: Rev. Mod. Phys. 88 (2016), p. 015003. DOI: 10.1103 /RevModPhys . 88.015003. arXiv: 1507.05893 [physics.ins-det]. 